\title{
Estudio sobre percepción de valores éticos en un proyecto de desarrollo tecnológico
}

\author{
A study on the perception of ethical values in \\ a technological development project
}

\section{Palabras clave}

Ética, desarrollo tecnológico,

investigación, participación usuarios, conciencia ética.

\section{Keywords}

Ethics, technological development, research, users' involvement, awareness of ethics.

\section{Introducción}

En este artículo desarrollamos un estudio acerca de las percepciones de los diferentes grupos participantes en una investigación en el campo de desarrollo de tecnología digital en referencia a su grado de involucración y sus conocimientos éticos. También estudiamos la comprensión de aspectos éticos por parte de estos grupos participantes, así como las expresiones de sus sentimientos y otros factores emocionales durante el proceso de investigación y sus reacciones frente a los resultados experimentales y la fase posterior a la propia experiencia. Por último, tratamos de proponer una serie de recomendaciones con el fin de ver si ciertas acciones proactivas beneficiarán la comprensión de las cuestiones éticas entre los grupos implicados.

La investigación que aquí se presenta exploró, por tanto, la necesidad de adquirir conciencia ética entre todas las partes interesadas en proyectos de investigación tecnológica. El proyecto al que hacemos referencia, APSIS 4 all (Sainz de Salces, F. y Bustamante Donas, J., 20I4), prestó una particular atención a la superación de las barreras de accesibilidad a las que
Fausto Javier Sainz de Salces

<faustosainzsalces@gmail.com>

SAPALA Consulting Ltd.

\section{Javier Bustamante Donas}

Departamento de Filosofía del Derecho, Moral y Política II.

Universidad Complutense de Madrid
Para citar:

Sainz de Salces, F. J. y Bustamante Donas, J. (20I6): "Estudio sobre percepción de valores éticos en un proyecto de desarrollo tecnológico". Revista Española de Discapacidad, 4 (2): 24I-25I.

Doi: <https://doi.org/IO.5569/23405 IO4.04.02.I $4>$ 
se enfrentan aquellas personas no familiarizadas con las TIC, personas con diversidad funcional y personas mayores, a la hora de interactuar con terminales digitales públicos, tales como cajeros automáticos y máquinas expendedoras de billetes de viaje. Como parte de la investigación se exploraron las cuestiones éticas planteadas en el uso de las tecnologías digitales, así como la toma de conciencia sobre aspectos éticos entre las partes interesadas.

En este informe se presentan los resultados de la investigación de campo llevada a cabo sobre cuestiones éticas. Al inicio del proyecto APSIS $_{4}$ all $^{\mathrm{r}}$ se identificó la necesidad de investigar acerca de la conciencia ética entre los sujetos involucrados en el proyecto. Como parte de los aspectos jurídicos y éticos del proyecto se llevó a cabo una investigación sobre cuestiones éticas. La investigación prestó una atención especial a la validez y suficiencia de la información proporcionada en una guía que también incluyó preguntas de sensibilización de carácter ético. Durante las sesiones de pruebas llevadas a cabo, en los tests se presentó un cuestionario a los voluntarios. Otro cuestionario fue entregado a los desarrolladores y al personal del proyecto APSIS4all. También se animó a expresar preocupaciones éticas, tanto al personal que trabaja en el proyecto como a los voluntarios que participan en los ensayos. Los resultados mostraron que la información proporcionada en la documentación era adecuada, y que tanto los voluntarios como el personal del consorcio eran conscientes de los derechos de los voluntarios y de las obligaciones del personal.

Es indiscutible que la tecnología y los valores mantienen una relación de mutua relevancia (Albrechtslund, 2007) y que muchos de los valores de la tecnología se reconocen en el debate académico al igual que la tecnología la propia evidencia y perfección de los valores éticos. La tecnología marca los limites de lo que podemos hacer; la ética, por su parte, delimita el campo

I. La investigación presentada en este documento se inscribe en el proyecto de investigación APSIS 4 all y del proyecto FFI 20I3-46908-R: "Ciencia, tecnología y sociedad: problemas políticos y éticos de la computación en nube como nuevo paradigma socio-técnico". de lo que debemos hacer. Ya que solamente se puede deliberar acerca de aquello que se puede hacer, queda claro que la relación entre ética y tecnología resulta intrínseca y esencial, inseparable desde cualquier punto de vista. Cuanto mayor es el poder de la tecnología, más necesario resulta la aplicación de un principio de responsabilidad que evalúe la adecuación de las realizaciones tecnológicas a fines socialmente relevantes, y no sólo a factores de relevancia científica. Sin embargo, aunque existe una innegable sensación sobre esta necesidad de incorporar valores éticos en los enfoques para el desarrollo de tecnología, en la práctica parece que no hay una voluntad decidida para satisfacer esta necesidad, ya que la ética se percibe frecuentemente como una limitación a las posibilidades de la propia tecnología. Y a los beneficios que ésta aporta. No obstante, ya existen ciertos procedimientos bien establecidos que se ajustan a principios básicos sobre ética, como el consentimiento informado o directrices y códigos de conducta profesional. Una excepción a esto es la Teoría del diseño sensible a valores (Friedman, I997) que propugna tener en cuenta los valores humanos como un factor esencial inherente a los procesos de innovación y desarrollo tecnológicos. Sin embargo, esta teoría es, por desgracia, desconocida en muchos campos del desarrollo tecnológico.

Esta necesidad de avanzar en las cuestiones éticas podría, por ejemplo, en última instancia, ser entendida a la luz del darwinismo ortodoxo que en un principio se describe por T.H. Huxley en su libro La evolución de la ética o bajo una luz más rousseauniana. Sin embargo, nuestra intención es más humilde: tratar de entender qué beneficios puede proporcionar un enfoque integral de desarrollo de la tecnología desde el punto de vista de los que se benefician directamente de ella, dejando de lado las consideraciones de una comprensión más profunda de la naturaleza humana, que incluyen sus orígenes y motivaciones, sean freudianas, o con origen en Lorenz o Darwin.

Sólo podemos esperar que el hecho mismo de la investigación llevada a cabo en este proyecto entre las partes interesadas y los participantes de 
la prueba haya servido, de alguna manera, como primera toma de contacto con las cuestiones éticas. Un contacto que con el tiempo puede, a través de la reflexión, ayudar a acercar los valores morales al diseño, según lo propuesto por Manders-Huits (2010). De algún modo es necesario legitimar la financiación de la investigación empírica sostenida sobre principios éticos apoyando y dando sustancia a estos valores.

Como prueba de la importancia de las cuestiones éticas podemos hacer referencia al hecho de que la Comisión Europea en su comunicación sobre Envejecer mejor en la sociedad de la información (2007) ha reafirmado sus puntos de vista y determinación sobre cuestiones éticas como un factor importante de inclusión digital. La investigación también exploró las percepciones acerca de los derechos que se encuentran establecidos en las leyes europeas, y entre esos derechos los que se refieren el respeto a la vida privada, el derecho a participar en la sociedad de la información y la protección de los datos personales (Mordini et al., 2009).

Otro efecto secundario de la investigación es la conciencia que los desarrolladores y los interesados pueden tener sobre las cuestiones éticas relacionadas con el desarrollo llevado a cabo, como ha señalado Jiménez (2008). En este caso, estamos de acuerdo en que durante la formación de los investigadores es importante para ellos ser conscientes de la responsabilidad de sus actos y que deben tener muy claro que no todo lo técnicamente posible es éticamente aceptable. Como señaló Vallor (20IO), tenemos que ser muy prudentes en el desarrollo de nuevas tecnologías para ser capaces de ver el significado ético de los nuevos desarrollos, con toda su complejidad, novedad y mutabilidad, y responder adecuadamente a posibles desafíos.

Como parte de los esfuerzos para entender la concienciación de los participantes en el proyecto sobre cuestiones éticas -sus percepciones, actitudes y conocimientosdurante el proceso de desarrollo tecnológico, dos cuestionarios fueron desarrollados con el fin de aplicarse a todos los socios y empleados involucrados en el proyecto, y los participantes en las actividades de evaluación. El primer cuestionario fue entregado a los que trabajaban en el proyecto para evaluar el nivel de conciencia ética entre los socios del proyecto, y también para investigar ciertos problemas en cuanto a su percepción acerca del grado de conciencia de los voluntarios.

Esperamos que esta acción contribuya, de alguna manera, a desarrollar un sentido de responsabilidad ética entre los desarrolladores, o al menos a considerar el efecto que una persona involucrada en un proyecto como desarrollador puede tener en el resultado final y sus implicaciones éticas. Tratamos de promover la consciencia de la responsabilidad ética existente cada vez que se desarrolla tecnología como ha sido propuesto por Burri Gram-Hansen (2009). Esta toma de conciencia debe estar inspirada desde el comienzo del proyecto y no una vez que la tecnología ya se ha desarrollado.

Otro cuestionario se pasó a los voluntarios durante la fase de pruebas (evaluación del prototipo). Este último cuestionario explora la adecuación de las cuestiones éticas durante la prueba y el seguimiento de los socios de las recomendaciones relativas a normas éticas.

La confianza en la tecnología es otro tema que teníamos la intención de tener en cuenta, sobre todo en el desarrollo de sistemas digitales que sean inclusivos en su diseño.

\section{Objetivos}

Los principales objetivos de la investigación sobre cuestiones éticas fueron los siguientes:

- Saber si las directrices previamente presentadas fueron percibidas como adecuadas.

- Evaluar el grado de conciencia personal sobre cuestiones éticas en el proyecto. 
- Obtener información sobre el cumplimiento de las normas éticas por parte de los investigadores, desarrolladores y trabajadores del proyecto.

- Explorar la percepción del personal investigador sobre los derechos de los participantes, la presión de grupo en relación con los avances tecnológicos y las medidas de sensibilización, así como las medidas paliativas en el caso de que un sujeto se retire de la investigación.

- Analizar los temas relacionados con la motivación y la decepción en los participantes.

\section{Metodología}

Con el fin de investigar las cuestiones planteadas anteriormente, se prepararon dos cuestionarios: un cuestionario destinado a los desarrolladores y personas que trabajan en el proyecto (personal del proyecto) y otro cuestionario dirigido a los voluntarios. Al personal del consorcio se le pasó el cuestionario después de presentarles el documento ético y las directrices legales con tiempo suficiente para leerlo previamente.

El segundo cuestionario, dirigido a los voluntarios, se les presentó después de que las actividades de evaluación hubiesen tenido lugar. Durante las sesiones los participantes siguieron los procedimientos estándar: primero se proporcionó información sobre el procedimiento a los voluntarios, quienes pudieron optar por continuar con la sesión mediante la firma de un consentimiento informado o por abandonar e irse. Dejando a un lado las posibles circunstancias que rodean el interés personal y la posible presión de los compañeros (Jansson et al. 2008), los que aceptaron participar y que habían firmado el documento de consentimiento informado luego realizaron las tareas y respondieron a los diferentes cuestionarios que conformaron toda la sesión de evaluación. Una vez terminado todo el procedimiento se les agradeció su participación, recibieron una gratificación y fueron informados de que si querían conocer los resultados de la investigación se les informaría al respecto. Los datos fueron analizados, y los resultados se presentan como estadísticas descriptivas.

\section{Resultados encuesta desarrolladores}

Los resultados del análisis estadístico de los datos recopilados de ambos cuestionarios durante la investigación se presentan en esta sección. En primer lugar, se presenta el análisis de los datos procedentes de los participantes pertenecientes a las instituciones que conforman el consorcio. Esta información será seguida por el análisis de los datos de los voluntarios en la prueba piloto.

\subsection{Conclusiones provisionales}

La respuesta afirmativa a la primera pregunta, ¿Sabía usted acerca de la necesidad de garantizar el cumplimiento ético y legal en el momento del inicio del proyecto?, implica la conciencia genérica sobre cuestiones jurídicas y éticas del personal que trabaja en el proyecto. También es importante tener en cuenta la realidad en cuanto a la dificultad percibida por los usuarios en materia de protección de datos y privacidad en relación con la legislación. El rápido desarrollo de la tecnología plantea un enorme desafío en este aspecto, donde a veces la legislación es contradictoria o los desarrolladores tienen que guiarse por legislaciones provenientes de otras áreas tecnológicas, que necesitan ser adaptadas ad hoc y podrían no cumplir con todos los requisitos sociales, personales y comerciales de esta área en particular.

Es relevante también destacar la percepción acerca de los beneficios de los avances tecnológicos entre los no tan jóvenes. Y en relación con la responsabilidad percibida de los usuarios en el caso de los encuestados sobre actividades de co-diseño ésta comprendía 
la libertad inherente a los voluntarios en las actividades de ensayo y co-diseño. Los encuestados también eran conscientes de los derechos y procedimientos para garantizar la privacidad y la libertad de los voluntarios. Esto también se aplica a las cuestiones relacionadas con los voluntarios acerca del compromiso, o nivel de compromiso, con respecto a la investigación, donde también se dieron a entender las posibles soluciones.

Parece que la guía proporcionada al consorcio sobre cuestiones éticas fue considerada como satisfactoria, tanto en contenido y relevancia como en cercanía y claridad para entender la documentación. Sin embargo, tal vez la inclusión de una lista de documentos específicos, y cómo acceder a ellos, podría haber sido útil. Aparecieron ideas interesantes, aunque realmente estas ideas no habían sido innovadoras en relación al compromiso social: una respuesta fue que las personas mayores prefieren el servicio personal, y por lo tanto ven los avances tecnológicos tales como máquinas de autoservicio beneficiosas para la empresa o el banco en lugar de para el usuario final. Las medidas de contingencia fueron percibidas como procedimientos necesarios y paliativos.

La honestidad es una actitud importante al colaborar en investigación, especialmente relevante en referencia a la información proporcionada a los participantes. Aparecieron ideas sobre la gestión de la participación de los voluntarios en las pruebas y su colaboración. Ideas sobre cómo alentar y promover la participación. La percepción general de las personas mayores como grupo no es diferente de otros segmentos de la población: son igualmente dignos de confianza.

La mayoría de los encuestados pensaban que la documentación aportada es relevante y adecuada para el proyecto, fácil de entender y asimilar, pero había claras expectativas en cuanto a la aplicación de las normas éticas. Esta idea fue expresada en varias ocasiones. También la mayoría de los encuestados parecía ser consciente de su implicación ética en el proceso de investigación.

\section{Resultados del cuestionario para voluntarios}

En esta sección presentamos los resultados más relevantes de la encuesta.

Respecto a las cuestiones relacionadas con la idea de efecto beneficioso de los avances tecnológicos y del posible origen de estas ideas debemos decir que la mayoría de respuestas eran muy claras en su opinión de que la tecnología tiene el potencial de proporcionar importantes beneficios a las personas mayores y personas con discapacidad. Los encuestados indicaron, contundentemente, que la tecnología podría ser de gran utilidad para las personas con discapacidad, ya que puede ayudar en la realización de las tareas cotidianas. Algunos consideraron que todos los avances tecnológicos tienen el potencial de mejorar las vidas de las personas con discapacidad, ya que pueden adaptarse a las necesidades específicas de los usuarios. Los encuestados consideraron, en general, que la tecnología puede mejorar la accesibilidad mediante la mejora de la movilidad, la comunicación y el acceso a la información, y de este modo mejorar la calidad de vida de las personas y ayudarles a aprender.

Uno de los encuestados expresaba lo que percibía como claros beneficios de la tecnología de la siguiente manera: "Los avances tecnológicos permiten sistemas de comunicación alternativos, movilidad y mejorar la autonomía en las actividades de la vida diaria". Otro añadió: "Las opiniones de los usuarios son importantes. En el futuro será posible mejorar y facilitar la vida a las personas con discapacidad haciendo que la tecnología sea más fácil de aprender y usar”. Otro encuestado consideraba que mientras que la tecnología podría ayudar a las personas con discapacidad, sigue sin ser utilizada en todo su potencial y algunos desarrollos todavía tienen que ser implementados: "La tecnología es útil, pero todavía hay algunos avances que no se están aplicando, como la traducción automática de idiomas". 
Algunos encuestados señalaron una barrera psicológica potencial de absorción de la tecnología, especialmente entre las personas mayores, lo que significa que algunos usuarios todavía tendrían dificultades, debido a menudo a la mentalidad del usuario. Esto es destacado por un entrevistado que comentó lo siguiente: "Depende del individuo. Algunas personas con discapacidad confían en la tecnología, otros no lo hacen". Este comentario pone de relieve la importancia de la confianza y la comprensión que tenemos sobre la tecnología. En términos generales, como ha señalado Turilli (2007), tenemos que entender lo que significa para nosotros que los objetos se comporten de acuerdo con los principios éticos respaldados por nuestra sociedad, especialmente hoy en día, con la amplia difusión de los artefactos y aplicaciones altamente desarrollados.

\subsection{Conclusiones provisionales}

Se puede observar a partir de las respuestas al cuestionario que los encuestados consideraron que la información que se presentó a los voluntarios era adecuada sobre cuestiones éticas durante las sesiones de validación llevadas a cabo en APSIS4all. Esto apunta a una buena aplicación de la recomendación hecha por Magnusson y Hanson (2003) en relación con los principios éticos relacionados con los proyectos de tecnología de investigación y desarrollo: “es decir, el respeto a la dignidad humana, el valor del individuo y sus derechos fundamentales, la autonomía y la privacidad, la confidencialidad, el consentimiento informado, el principio de no maleficencia, justicia, beneficencia, y veracidad".

No aparecieron preocupaciones éticas, por parte de los voluntarios, relacionadas con las pruebas realizadas, por lo tanto la información proporcionada en el consentimiento informado y durante la sesión de prueba debe haber sido adecuada al confirmarse el cumplimiento previo de las indicaciones provistas en la guía ética por parte de los evaluadores.

Todos los participantes pensaron que fueron adecuadamente informados sobre sus responsabilidades, y que el consentimiento informado incluyó toda la información esperada. Sin embargo, se podría argumentar que los participantes no expresaron su oposición al consentimiento informado porque no lo entendieron. En este sentido, ya que los participantes no tenían ninguna patología que limitase su comprensión, tenemos que entender estas respuestas como el resultado de un documento bien presentado con información clara sobre los derechos de los participantes en la prueba (Löfman, 2004).

La mayoría de los voluntarios estuvieron de acuerdo con la idea de que los desarrollos tecnológicos ayudan a las personas con discapacidad y las personas mayores. Aunque también hubo comentarios críticos sobre este punto de vista.

\section{Conclusiones}

El documento de referencia que se presentó a los participantes sobre aspectos éticos incluía información acerca de la necesidad de un documento que explicitase el consentimineto del participante en las evaluaciones (consentimiento informado), información acerca de los objetivos del proyecto y del procedimiento de evalaucion, de la compensación a recibir, necesidad de cumplir con las norms europeas sobre privacidad y seguridad, necesidad de evitar riesgos o malestar durante las sesiones, tener la oportunidad de cuestionar o preguntar acerca de aspectos éticos del procedimiento antes, durante y después de que este se llevase a cabo, etc ${ }^{2}$. Aunque no es posible demostrar la mejora en adquisición de conciencia ética por medio de la información presentada al inicio del proyecto, mediante este documento se observó un claro avance en esta toma de conciencia.

2. Para más informacion ver: Sainz de Salces, F. y Bustamante Donas, J. (20I4): “Aspectos éticos en la participación de personas con diversidad funcional en proyectos de investigación tecnológica”. Revista Española de Discapacidad, 2 (I): I65-I74. 
Parte de este avance se desarrolló a través de la elaboración y presentación de las directrices a los participantes. En general, las principales conclusiones del estudio fueron:

- La guía presentada sobre cuestiones éticas y legales era adecuada y cumplió sus objetivos. Sin embargo, incluir información detallada sobre recursos puede ser un buen paso para mejorar la documentación. Una aclaración sobre la forma de proceder con respecto a la implementación de las directrices éticas podría ser necesaria en ciertos casos.

- La mayoría de los voluntarios y trabajadores del proyecto son conscientes de los derechos de los participantes de la prueba, por lo tanto, los derechos de los participantes parecen ser reconocidos por todas las partes interesadas.

- La percepción de los participantes sobre cuestiones éticas se equiparó a la percepción de los desarrolladores sobre la conciencia ética de los participantes.

- No hubo diferencias entre ambos grupos en cuanto a la variedad de puntos de vista en relación con el desarrollo tecnológico y sus efectos sobre las personas mayores y personas con discapacidad, lo que confirma los puntos de vista divergentes sobre el asunto.

Aunque el área de la ética en la tecnología no era familiar para la mayoría del personal de APSIS $_{4}$ all, la documentación aportada durante los primeros meses del proyecto en relación con las cuestiones éticas y legales parece haber ayudado a este grupo a familiarizarse con estos temas. Por los resultados de la encuesta parece que se ha logrado una concienciación de los socios y voluntarios en temas éticos. Es nuestra esperanza que al reflexionar sobre las cuestiones éticas planteadas a los desarrolladores estos se den cuenta de cómo la tecnología no debe ser considerada neutral, sino que a veces tiene repercusiones éticas y políticas en la vida de las personas (Manders-Huits, 20IO). Los autores esperan que este trabajo sepa promover el diálogo y el debate, especialmente en relación con los aspectos éticos de diseño de la tecnología para los menos favorecidos, teniendo en cuenta los nuevos paradigmas, como la computación en nube y la robótica en un mundo que cambia rápidamente, y donde la tecnología parece llegar muy por delante de los valores éticos. 
Anexo 1: Encuesta a desarrolladores

o. ¿Sabía usted acerca de la necesidad de garantizar el cumplimiento ético y legal en el momento del inicio del proyecto?

Sección A: Aspectos generales.

I. ¿Se siguieron las directrices y recomendaciones sobre aspectos éticos?

2. ¿Cree usted que los voluntarios son conscientes de sus derechos?

3. Muchos investigadores y jóvenes consideran que los avances tecnológicos suponen siempre una mejora en la calidad de vida. ¿Las personas mayores piensan lo mismo? ¿Quién o qué les han convencido de los beneficios de la tecnología? ¿Ha sido siempre una opinión generada de forma natural o han sido forzados a ello?

4. ¿Qué responsabilidades tienen los participantes en la experiencia de co-diseño?

5. ¿Qué pasa con los derechos de propiedad intelectual cuando los voluntarios están proporcionando ideas y resultados?

6. ¿Cuál es el enfoque ético correcto cuando un sistema tiene la capacidad de controlar una gran cantidad de cosas, desde el movimiento en habitaciones particulares a si una persona ha tomado su medicina, $y$ de este modo recopila información sobre los hábitos y las condiciones diarias de una persona, y luego la transmite en tiempo real a los médicos o miembros de la familia?

Sección B: Compromiso.

7. Si los participantes no pueden asistir a la sesión de trabajo la interrupción causada por su comportamiento podría, de hecho, causar problemas en la agenda de investigación, etc., además de otras posibles implicaciones. Entonces, ¿cómo se puede abordar este problema?

8. ¿No es un comportamiento que deba ser reprendido?
9. ¿Es apropiado dejarlos ir "impunes"?

ı。. ¿Cómo podemos confiar en las personas mayores participen en los experimentos y cumplir con sus compromisos?

I I. ¿Cuáles son las implicaciones morales de no poder asistir a una sesión de co-diseño?

I2. ¿Qué puede hacer un equipo de investigación para hacer frente a una serie de ausencias continuas de los participantes? ¿Se trata solamente de que los investigadores tienen que prever este tipo de comportamiento (incapacidad de los participantes para cumplir con los compromisos de investigación) y actuar en consecuencia?

I3. ¿Qué sucede con los participantes cuando el proyecto está terminado y se les retira la tecnología? ¿Qué deben hacer los investigadores para asegurar que los participantes no queden decepcionados, frustrados o infelices al final del proyecto?

I4. ¿Qué sucede con las personas y sus deseos y esperanzas, cuando el desarrollo del proyecto está estancado o terminado, y los servicios tecnológicos utilizados, que se prometieron o experimentaron, son retirados?

Sección C: Documentación e información. Cuestiones éticas.

I 5. ¿Cree usted que la documentación aportada es relevante para el proyecto?

I6. ¿Los contenidos del documento se adecuan al proyecto?

I7. ¿Fueron los objetivos de documento claramente establecidos al principio de este?

I8. ¿Fue fácil aproximarse al contenido?

I9. ¿Fue fácil de entender?

20. Indique en la siguiente escala, el nivel al que se siente los objetivos se han alcanzado (donde $\mathrm{I}=\mathrm{IO} \%$ y $\mathrm{IO}=\mathrm{I} 00 \%$ ).

2I. ¿Qué se podría haber hecho o qué siente que podría haber facilitado la adquisición de información en el área ética? 
22. ¿Hubo algún inconveniente para seguir los procedimientos éticos? Si has encontrado algunos obstáculos para seguir las directrices éticas, ¿cuáles fueron?

Sección D: Experiencia de los participantes.

Para medir cómo los encuestados se sienten sobre el esfuerzo realizado para que los colegas tomen conciencia acerca de las cuestiones éticas se utilizó una escala Likert, donde I es el valor asignado para representar una tarea muy fácil y 5 representa una tarea percibida como muy difícil.
La pregunta estaba redactada de la siguiente manera:

23. Hacer que los colegas tomen conciencia de las cuestiones éticas y de su necesidad de cumplimiento era:

24. ¿Se dan cuenta los voluntarios de las implicaciones éticas de su participación en la investigación?

25. ¿Se dan cuenta los voluntarios de las posibles implicaciones éticas en el desarrollo de productos y sobre las consecuencias de su uso?

26. ¿Comentaron los voluntarios sobre cualquier aspecto ético durante el tiempo de la investigación? 
Anexo 2: Encuesta a voluntarios

Perfil demográfico de los encuestados:

Género: $53 \%(\mathrm{n}=\mathrm{I} 6)$ de los encuestados eran mujeres, $47 \%(n=14)$ eran hombres. Edad: todos menos uno de los encuestados tenían entre 30 años o superior, desde los 28 a 73 años de edad y siendo la edad media de 48,3 .

Perfil: los encuestados indicaron que tenían experiencia en toda una gama de categorías de discapacidad. Debe tenerse en cuenta que se hizo una distinción entre las personas sordas y con problemas de audición para que coincida con las respuestas de los encuestados.

Sección A: Completada antes de las pruebas de usuario.

I. Con la explicación que has recibido sobre el proyecto y la prueba que estás a punto de realizar, y tras leer el consentimiento informado ¿te han quedado claros cuáles son tus derechos como participante?

2. ¿Conocías estos derechos?

3. ¿Conoces tus responsabilidades como participante en esta prueba que estás a punto de realizar?

4. ¿Crees que se te ha informado adecuadamente de tus responsabilidades?

5. El formulario de consentimiento informado que has firmado ¿aportaba toda la información que esperabas/deseabas?

6. ¿Existe algún asunto de carácter ético que te preocupe en relación con el proyecto?
7. ¿Has participado con anterioridad en pruebas similares o en otros proyectos de investigación?

8. En caso de haber participado en otras pruebas ¿crees que te informaron correctamente de tus derechos y responsabilidades?

9. Existe la idea general de que los avances tecnológicos conllevan una mejora de la calidad de vida de las personas, y especialmente de las personas con discapacidad y mayores.

a. Se pidió a los participantes que calificaran subjetivamente su acuerdo con esta afirmación en una escala Likert de I-7; Siendo I totalmente en desacuerdo y 7 muy de acuerdo.

b. ¿Por qué crees que las personas con discapacidad y las personas mayores aceptan en principio la idea de efecto beneficioso de los avances tecnológicos? ¿Crees que es una opinión personal o es algo generado por los medios de comunicación o por los investigadores y tecnólogos?

Sección B: Esta sección fue rellenada tras realizar las pruebas.

Io. ¿Hay algún aspecto ético que te preocupe sobre la prueba que acabas de realizar? 


\section{Referencias bibliográficas}

Albrechtslund, A. (2007): "Ethics and technology design". Ethics and information technology, 9: $63-72$.

Burri Gram-Hansen, S. (2009): Towards an Approach to Ethics and HCI Development Based on Løgstrup's Ideas. Lecture Notes in Computer Science, 5726, 200-203.

Comisión Europea (2007): Ageing well in the information society: action plan on information and communication technologies and ageing, an i2010 initiative. En Communication from the Commission to the European Parlament, the Council, the European Economic and Social Committee of the Regions. Bruselas.

Friedman, B. (1997): Human Values and the Design of Computer Technology, Stanford, California \& Cambridge, New York: CSLI Publications \& Cambridge University Press.

Jansson, M., et al. (2008): Participation in e-Home Healthcare@North Calotte. En Proceedings NordiCHI 2008. Lund.

Jiménez Garrote, J. L. (2008): “Aspectos éticos a tener en cuenta en la investigación psicofarmacológica”. Bioética, 6 (I): I 8-2I.
Löfman, P. et al. (2004): "Ethical issues in participatory action research". Scandinavian Journal of Caring Sciences, I 8: 333-340.

Magnusson, L. y Hanson, E. J. (2003): "Ethical issues arising from a research, technology and development Project to support frail older people and their family carers at home". Health and Social Care in the Community, I I (5): 43 I-439.

Manders-Huits, N. (2010): "What values in design? The challenge of incorporating moral values into design". Science and Engineering Ethics, I7 (2): 27I-287.

Mordini, E. et al. (2009): "Senior citizens and the ethics of e-inclusion". Ethics Information Technology, I I: 203-220.

Sainz de Salces, F. J. et al. (20I4): "Involucración de personas con discapacidad en proyectos tecnológicos de I+ D+ i: el caso de APSIS4all”. Revista Española de Discapacidad, 2 (2): I 2 II 44 .

Turilli, M. (2007): "Ethical protocols design". Ethics and Information Technology, 9: 49-62.

Vallor, V. (2010): “Social networking technology and teh virtues". Ethics and Information technology, I2: I 57-170. 